\title{
Strongyloides stercoralis hyperinfection in a patient with angioimmunoblastic lymphadenopathy
}

\author{
Ahmad K. Uwaydah and Farouq Al Qadah \\ Department of Medicine, Hamad Medical Corporation, PO Box 3050, Doha, Qatar
}

\begin{abstract}
Summary: A 29 year old Bengali male patient on chemotherapy for angioimmunoblastic lymphadenopathy developed Strongyloides hyperinfection syndrome 3 months after being treated with a single 3 day course of thiabendazole. His complicated hospitalization and successful management are described. Prevention of this potentially fatal disease in immunocompromised patients by early diagnosis and proper management of intestinal strongyloidiasis is emphasized.
\end{abstract}

\section{Introduction}

Strongyloides stercoralis hyperinfection syndrome, well recognized since $1966,{ }^{1}$ has increased in frequency during the past decade as a result of immunosuppressive therapy used in the treatment of organ transplant recipients, collagen vascular diseases and cancer. We describe herein a patient with angioimmunoblastic lymphadenopathy who developed Strongyloides hyperinfection 3 months following thiabendazole therapy of his intestinal strongyloidiasis.

\section{Case report}

Our patient is a 29 year old Bengali male who presented in August 1990 with weight loss, intermittent fever and night sweats of 8 months duration. Physical examination revealed generalized lymphadenopathy, his liver and spleen were not enlarged, and his routine laboratory investigations were non-contributory. He was diagnosed to have angioimmunoblastic lymphadenopathy based on right epitrochlear lymph node biopsy that showed total obliteration of the lymph node architecture with cellular proliferation and abundant arborizing blood vessels, the cellular component consisting of plasma cells, lymphocytes, immunoblasts, eosinophils and macrophages. ${ }^{2}$

He initially received six courses of doxorubicin, vincristine and prednisone. However, due to tumour progression, chemotherapy was changed to daily 6-mercaptopurine and prednisone in March 1991. This resulted in partial remission and chemotherapy was stopped after 2 months.

Correspondence: Ahmad K. Uwaydah, M.D.

Accepted: 11 May 1992
He presented 3 weeks later with history of abdominal pain, nausea and vomiting of 2 weeks duration. Physical examination revealed no significant abdominal findings, however, several lymph nodes were noted to have increased in size. Stooh examination disclosed the presence of Strongy loides stercoralis larvae and thiabendazole $1.0 \mathrm{~g}$ twice daily for 3 days was administered, 6-mercaptopurine and prednisone were resumed and his abdominal symptoms improved. However, he was admitted 3 months later because of severe shortness of breath for 1 day associated with dry cough, fever and abdominal pain. His chest X-ray showed diffuse bilateral reticulonodular pattern. Bronchoscopy revealed widespread bronchial mucosal blood oozing. Larvae of $S$. stercoralis were demonstrated in the bronchial wash and stool specimens. In addition, bronchial wash cultures gave a profuse growth of Pseudomonas aeruginosa.

The patient was placed on mechanical ventilation, given broad spectrum antibacterial therapy (imipenem/cilastatin) and thiabendazole $1 \mathrm{~g} \mathrm{(25}$ $\mathrm{mg} / \mathrm{kg}$ ) twice daily via a nasogastric tube. Tracheal aspirate taken on the seventh day of therapy with thiabendazole showed persistent $S$. stercoralis larvae, which cleared on repeat examination as the course of thiabendazole was extended to 2 weeks in total. His hospital course was further complicated by Candida tropicalis fungaemia that was treated with a course of amphotericin B and Staphylococcus aureus subclavian catheter sepsis that responded to intravenous vancomycin.

He was discharged with a clear chest X-ray after 6 weeks hospitalization. He was seen in the outpatient clinic one week after discharge, he appeared weak and his inguinal lymph nodes were grossly enlarged indicating reactivation of his original 
disease. Because of the poor general condition, no further chemotherapy was offered.

\section{Discussion}

The association between angioimmunoblastic lymphadenopathy and opportunistic infections related to suppressed T-cell function is well established. ${ }^{3,4}$ This is well exemplified in this case as Strongyloides stercoralis is one of the opportunistic pathogens in patients with impaired cellular immunity. In addition, Strongyloides can persist in a dormant state in the host's intestine for several years waiting to flare up when the host's cellular immunity decreases as a result of immunosuppressive drugs and/or diseases. ${ }^{5}$ Therefore it is essential for oncologists to be aware of this entity, especially when committing patients coming from endemic areas to systemic chemotherapy. Strongyloides hyperinfection, a potentially fatal disease that carries almost $86 \%$ mortality rate $^{6}$ is preventable provided a high index of

\section{References}

1. Cruz, T., Reboucas, G. \& Rocha, H. Fatal strongyloidiasis in patients receiving corticosteroids. $N$ Engl $J$ Med 1966, 275: 1093-1098.

2. Jaffe, E.S. Morphologic features, pp. 577-579. In: Steinberg, A.D. (moderator) Angioimmunoblastic lymphadenopathy with dysproteinemia. Ann Intern Med 1988, 108: 575-584.

3. Seldin, M.F. Clinical description, pp. 575-577. In: Steinberg, A.D. (moderator) Angioimmunoblastic lymphadenopathy with dysproteinemia. Ann Intern Med 1988, 108: 575-584.

4. Knecht, $H$. Angioimmunoblastic lymphadenopathy: ten years experience and state of current knowledge. Semin Hematol 1989, 26: 208-215.

5. Devault, G.A., King, J.W., Rohr, M.S., Landrenean, M.D., Brown, S.T. III \& McDonald, J.C. Opportunistic infections with Strongyloides stercoralis in renal transplantation. Rev Infect Dis 1990, 12: 653-671. suspicion is maintained and patients suspected to have chronic, persisting $S$. stercoralis infection are thoroughly investigated and treated before undergoing immunosuppressive therapy.

Our patient was treated with a 3 day thiabenazole course, a regimen reported to be effective in eradicating intestinal strongyloidiasis in $90-100 \%$ of patients. ${ }^{6}$ However, he succumbed to the hyperinfection syndrome 3 months later. This supports the recommendation that, because of the difficulty in confirming eradication, a repeat 2-day course of thiabendazole therapy one week after the initial course should be administered. ${ }^{7}$ Thiabendazole, to date, remains the treatment of choice for all forms of strongyloidiasis. ${ }^{8}$ Ivermectin has been shown in one study to be equivalent to thiabendazole in the treatment of strongyloidiasis; however, its role needs to be further defined. ${ }^{9}$ For the hyperinfection syndrome, a 2 week course of thiabendazole with frequent examination of stool, sputum or duodenal secretions seems adequate.

6. Igra-Siegman, Y., Kapilia, R., Sen, P. et al. Syndrome of hyperinfection with Strongyloides stercoralis. Rev Infect Dis 1981, 3: 397-407.

7. Pearson, R.D. \& Guerrant, R.L. Strongyloides infections. In:을 Strickland, G.T. (ed.) Hunter's Tropical Medicine. W.B. Saunders Company, Philadelphia, 1991, pp. 706-711.

8. Grove, D.I. Treatment of strongyloidiasis with thiabendazole: an analysis of toxicity and effectiveness. Trans $R$ Soc Trop Med Hyg 1982, 76: 114-118.

9. Naquira, C., Jimenez, G., Guerra, J. et al. Ivermectin for human strongyloidiasis and other intestinal helminths. Am J Trop Med Hyg 1989, 40: 304-309. 